\title{
Inhibidores de puntos de control inmunitario en estadios avanzados de melanoma
}

\author{
Immune checkpoint inhibitors in advanced stages of melanoma
}

\author{
Arely Fernanda Tamariz Campillo, ${ }^{*}$ Axel Corona Deschamps, ${ }^{*}$ Raúl Mellado Orellana,* \\ Enrique Juan Díaz Greene, ${ }^{\ddagger}$ Federico Leopoldo Rodríguez Weber ${ }^{\S}$
}

Citar como: Tamariz CAF, Corona DA, Mellado OR, Díaz GEJ, Rodríguez WFL. Inhibidores de puntos de control inmunitario en estadios avanzados de melanoma. Acta Med. 2021; 19 (1): 108-115. https://dx.doi.org/10.35366/98579

\section{Resumen}

El melanoma es el cáncer de piel con mayor mortalidad en el mundo, gracias a su alto potencial metastásico. En los últimos años se ha visto un aumento en su incidencia de manera global, incluyendo a México. Muchos son los mecanismos involucrados en la patogenia del melanoma, por lo que ha sido difícil el desarrollo de terapias específicas para su curación. Sin embargo, desde hace algunos años la inmunoterapia ha ido retomando interés en el melanoma, gracias a la introducción de los inhibidores de los puntos de control inmunitario. Estos fármacos han logrado de manera significativa mejorar la supervivencia a largo plazo de pacientes con melanoma avanzado. Desde entonces, la comunidad científica se ha enfocado en reconocer sus efectos y comparar su eficacia con tratamientos previamente utilizados, que hasta el momento no habían logrado obtener los mismos resultados. Desafortunadamente, cierto número de pacientes continúa sin lograr una respuesta satisfactoria, sin olvidar los múltiples efectos adversos inmunitarios asociados. En este artículo de revisión se describen los resultados de los estudios más recientes realizados con inhibidores de puntos de control, las dosis recomendadas y la frecuencia de sus efectos adversos inmunológicos.

Palabras clave: Melanoma, BRAF, oncogenes, CTLA-4, ipilimumab, PDL-1, nivolumab, pembrolizumab.

\section{Abstract}

Melanoma is the type of skin cancer with highest mortality worldwide due to its metastatic potential. In the recent years there has been an increasing incidence of melanoma worldwide, including in Mexico. There are several mechanisms involved in the pathogenesis of melanoma making it difficult to develop specific therapies. However, recently immunotherapy has been taking interest in treatment of melanoma, due to the introduction of inhibitors of immune checkpoints, this type of therapy has significantly improved long-term survival in patients with advanced melanoma. Therefore, the scientific community has focused on comparing side effects and efficacy versus standardized treatments, until today no better outcomes have been found, on the contrary patients can present an immune related response due to their action mechanism. Unfortunately, only a few patients have shown a satisfactory response to this type of therapy. This article reviews the results of the most recent studies conducted with checkpoint inhibitors, recommended doses, and frequency of adverse effects on immune system.

Keywords: Melanoma, BRAF, oncogenes, CTLA-4, ipilimumab, PDL-1, nivolumab, pembrolizumab.
* Residente de Medicina Interna.

‡ Profesor Titular de Curso de Medicina Interna.

$\S$ Profesor adjunto del Curso de Medicina Interna.

Hospital Ángeles Pedregal. Facultad Mexicana de Medicina de la Universidad La Salle. Ciudad de México, México.

Correspondencia:

Dr. Arely Fernanda Tamariz Campillo

Correo electrónico: aftamariz7@gmail.com

Aceptado: 03-08-2020.

www.medigraphic.com/actamedica

\author{
Abreviaturas: \\ BRAF = Gen que codifica para una proteína implicada en la \\ señalización del receptor del factor de crecimiento epidérmico. \\ $\mathrm{FDA}=$ Food and Drug Administration. \\ CTLA-4 = Antígeno-4 asociado al linfocito T citotóxico. \\ PD-1 = Receptor de muerte programada 1. \\ NRAS $=$ Homólogo del oncogén viral RAS de neuroblastoma.
}

Abbreviations:

$\mathrm{BRAF}=\mathrm{B}-$ Raf proto-oncogene, serine/

threonine kinase.

$\mathrm{FDA}=$ Food and Drug Administration.

CTLA-4 = Cytotoxic T-lymphocyte-associated protein-4.

PD-1 = Programmed death protein 1.

NRAS $=$ Neuroblastoma RAS viral oncogene homolog.

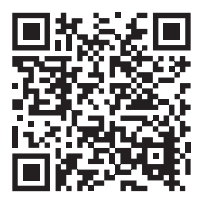




\section{HISTORIA}

En el 2018, el Premio Nobel de Medicina fue otorgado a James P. Allison y a Tasuku Honjo por la terapia contra el cáncer mediante la inhibición de la regulación inmune negativa, específicamente por el desarrollo de dos nuevos fármacos, un inhibidor de la proteína CTLA-4 (antígeno-4 asociado al linfocito T citotóxico) e inhibidor de la proteína PD-1 (receptor de muerte programada-1), que fueron comercializados a principios del 2011 cambiando el paradigma del melanoma en etapas avanzadas. ${ }^{1}$ Aunque estos descubrimientos sean sumamente recientes, la primera descripción de melanoma fue encontrada en los escritos de Hipócrates, donde la palabra melanoma proveniente de griego melas (obscuro) y oma (tumor), fue hallada por primera vez en el sigo 5 a.C. ${ }^{1}$ La primera evidencia encontrada en algún resto humano fue hallada en un esqueleto humano de la civilización precolombina mummies, en donde localizaron metástasis difusas en hueso, el estudio por radiocarbono reveló que el cuerpo databa desde hace por lo menos 2,400 años. ${ }^{1}$ El cirujano John Hunter, que laboraba en el hospital St. George's Hospital Medical School, en Londres, es reconocido por remover por primera vez un melanoma de la mandíbula de un hombre de 35 años en $1787 .{ }^{1}$ El tumor fue preservado y calificado como melanoma en 1968 y se encuentra en el museo de Hunterian en Lincoln's Inn Field. En 1858 Oliver Pemberton describió una serie de 60 casos de melanomas, así como sus diversas características, además de la localización más común de metástasis, y se describió el primer paciente afroamericano con melanoma. ${ }^{1}$ En 1956, Henry Lancaster describió la relación entre la exposición de luz ultravioleta en población caucásica: la intensidad y duración de la exposición estaba directamente relacionada con la incidencia de melanoma. ${ }^{1}$ En 1970 Alexander Breslow describe que el pronóstico del melanoma depende del estadio, pero principalmente de la invasión vertical del tumor, que posteriormente daría resultado al índice de Breslow. ${ }^{1}$ Desde el descubrimiento de los oncogenes asociados al melanoma a partir de 1980, el estudio de la biología molecular del melanoma se ha enfocado en el descubrimiento de mutaciones asociadas a dichos genes. ${ }^{1}$ Entre las mutaciones con mayor interés se encuentran la mutación NRAS (neuroblastoma $R A S$ viral oncogen homolog, por sus siglas en inglés) y BRAF, ambas asociadas al valor pronóstico del melanoma. En el caso de la mutación BRAF, predice una disminución en la supervivencia en el contexto de melanoma metastásico. ${ }^{1}$

\section{EPIDEMIOLOGÍA}

El melanoma maligno es un cáncer de piel potencialmente mortal, su incidencia ha ido en aumento en el ámbito mundial, particularmente en Estados Unidos, Europa y en países con población de predominio caucásico. ${ }^{2}$ Las tasas estimadas de incidencia de melanoma por edad en hombres y mujeres en todo el mundo aumentaron de 2.3 y 2.2 por cada 100,000 personas respectivamente durante 1990, a 3.1 y 2.8 por cada 100,000 en 2008. ${ }^{3,4}$ Aunque el melanoma es menos común que otros tipos de cáncer de piel, es el más letal y representa casi el $73 \%$ de muertes. ${ }^{2}$ De acuerdo con la American Cancer Society, el riesgo de contraer melanoma durante la vida es de aproximadamente $2.6 \%$ ( 1 en 38 ) para los blancos, $0.1 \%$ ( 1 en 1,000$)$ para los afroamericanos y $0.6 \%$ ( 1 en 167) para los hispanos. ${ }^{5}$ Hasta el 2018 en México, el melanoma de piel tuvo una incidencia de $1.6 \%$, lo cual representa un aproximado de 3,079 casos nuevos, con una prevalencia a cinco años de 9,081 casos. $^{6}$ La dirección general de salud reportó 1.2 defunciones por melanoma por cada 100,000 habitantes, y la clínica de melanoma del Instituto Nacional de Cancerología reportó un aumento de melanoma en los últimos años del $500 \%$, con una tendencia similar para este año. ${ }^{7}$

\section{ETIOLOGÍA Y PATOGÉNESIS}

El melanoma cutáneo es una neoplasia que inicia en la unión dermoepidérmica de la piel normal o en el sitio de un nevo melanocítico preexistente. De igual forma, el melanoma puede iniciarse en cualquier sitio no cutáneo, en donde normalmente habitan melanocitos; esto incluye superficies mucosas y tracto uveal, siendo estos últimos menos frecuentes para el desarrollo de melanoma. ${ }^{8}$ Los melanocitos se originan de las células derivadas de la cresta neural durante el desarrollo embriológico, colonizando la piel, ojos, y en menor grado otros tejidos del organismo. ${ }^{9}$

Su patogénesis involucra una serie de eventos endógenos y exógenos, todos éstos influenciados bajo una predisposición genética y exposición a luz ultravioleta, siendo este último el principal factor de riesgo para su desarrollo. El antecedente familiar ocurre en el $10 \%$ de los pacientes con melanoma; en las miembros de estas familias existe la mutación cinasa dependiente de ciclina 2A (CDKN24), que se encuentra entre el 24 a 40\% de los casos. Este gen codifica dos supresores tumorales (p16 y p14) y representa un vínculo directo para el desarrollo de melanoma. ${ }^{10}$ Otros genes se han relacionado con la patogénesis del melanoma, lo cierto es que en más del $50 \%$ de los melanomas cutáneos se desconoce su base genética. ${ }^{9}$

Los melanomas esporádicos representan aproximadamente el $90 \%$ de todos los casos de melanoma en los que se involucran mutaciones somáticas, expresadas por alelos con una prevalencia alta y penetrancia baja, lo que nos orienta a reconocer los factores ambientales como actor importante en la transformación maligna de los melanocitos. ${ }^{11-13}$ Las mutaciones somáticas más frecuentes en melanoma 
afectan genes en vías de señalización clave que lideran la proliferación (BRAF, NRAS y NF1), crecimiento y metabolismo (PTEN y KIT), identidad celular (ARID2), resistencia a apoptosis (TP53), control del ciclo celular (CDKN2A) y replicación celular (TERT). ${ }^{14-17}$ Por otro lado, en ocasiones el melanoma primario se asocia con lesiones precursoras que incluyen el nevo melanocítico común, nevo melanocítico displásico y nevo melanocítico congénito. ${ }^{18}$

\section{FACTORES DE RIESGO}

Los factores de riesgo para el desarrollo de melanoma los podemos dividir en factores de riesgo modificables y factores de riesgo no modificables. ${ }^{19}$ Los modificables son: exposición ultravioleta y fármacos. La luz ultravioleta es un claro factor de riesgo para desarrollo de melanoma, incluyendo las camas de bronceado. ${ }^{20,21}$ Múltiples estudios han demostrado que la exposición prolongada mayor a 20 años incluyendo la exposición ocupacional, aumenta de manera considerable el riesgo de desarrollo de melanoma. Sin olvidar que la exposición a rayos UV puede ocurrir durante procedimientos médicos, como en el caso de pacientes que reciben terapia con PUVA (psoralenos con radiación ultravioleta $\mathrm{A}$ ), el riesgo de melanoma aumenta a partir de 250 sesiones de tratamiento. ${ }^{22}$ Por otro lado, los factores de riesgo no modificables incluyen los componentes genéticos, estatus socioeconómico, raza, número de nevos, edad y género. ${ }^{19}$ La raza caucásica tiene 10 veces más riesgo de desarrollar melanoma cutáneo comparado con otras razas con fototipo mayor. Haciendo énfasis en que en la población no caucásica es más común el melanoma no cutáneo. Los hombres tienen 1.5 mayor riesgo de desarrollar melanoma, ${ }^{23,24}$ con una edad diagnóstica promedio de 57 años. Pero difiriendo según el género, de los 25-40 años es más común en mujeres, después de los 75 años es más común en hombres. ${ }^{23}$ En cuando al factor genético, el melanoma familiar se presenta en el $10 \%$ de los casos que presentan melanoma, la mutación encontrada más comúnmente en el melanoma familiar es la CDKN2A, un oncogén del tipo supresor tumoral. ${ }^{18}$ La presencia de nevos displásicos está asociado con un aumento de $1.5-10 \%$ de presentar melanoma, y depende del número de nevos displásicos presentes. ${ }^{25} \mathrm{El}$ riesgo de presentar melanoma es de 1.5 veces mayor en sujetos con nevos displásicos de 11 a 25, siendo el doble si existe un número mayor a $25 .{ }^{24}$ Las diversas causas de inmunosupresión, como los pacientes postrasplantados que requieren de diversos fármacos inmunosupresores, requieren de una valoración dermatológica anual para la detección temprana del cáncer, puesto que tienen un riesgo incrementado que va de 2-4 en comparación con quienes no la presentan. ${ }^{19}$
Los melanomas pueden dividirse según su histopatología en cuatro tipos principales: ${ }^{26}$

- Melanoma superficial diseminado (70\%): es el más común, puede localizarse en cualquier sitio anatómico; se caracteriza por un crecimiento radial antes que vertical (invasivo), con color desde marrón, negro, azul y rojo.

- Melanoma nodular (15 al 30\%): se origina como una masa con elevación uniforme o lesión polipoide; rápido crecimiento vertical desde su inicio, comúnmente azul o azul negruzco.

- Melanoma lentigo maligno (4 a 10\%): mayormente asociado al daño solar crónico en personas de edad avanzada; conforme evoluciona, se vuelve más obscuro, asimétrico con un fase de crecimiento vertical.

- Melanoma lentigo acral (<5\%): las lesiones se originan más frecuentemente en palmas y plantas, subungueales y ocasionalmente de superficies mucosas.

\section{PRONÓSTICO}

La supervivencia del melanoma cutáneo depende del estadio y extensión de la enfermedad al momento del diagnóstico. El factor pronóstico más importante del melanoma primario es el espesor del tumor, el cual se mide a través del índice Breslow, éste mide la invasión del tumor desde la epidermis hasta la porción más profunda del tumor. ${ }^{18}$ En general, los melanomas menores a $1 \mathrm{~mm}$ de espesor tienen muy baja probabilidad de diseminarse, con un índice de supervivencia a 10 años de 80 a 90\%; mientras que lesiones mayores a $4 \mathrm{~mm}$ presentan un índice de supervivencia de 40 a 50\% en 10 años. ${ }^{24}$ Otros factores asociados al pronóstico incluyen ulceración, índice mitótico y presencia de microsatélites. Asimismo, el involucro de los ganglios linfáticos regionales es un factor importante en la supervivencia. ${ }^{24}$ En la actualidad, se estima que la supervivencia a cinco años es aproximadamente del $98 \%$ para la enfermedad localizada (estadios 0, I y II); con el involucramiento de ganglios linfáticos regionales (estadio III), las tasas de supervivencia a cinco años son del $65 \%$, mientras que en enfermedad metastásica (estadio IV) la supervivencia a cinco años disminuye hasta en un $23 \% .27,28$

\section{INHIBIDORES DE PUNTOS DE CONTROL INMUNITARIO}

Para que la progresión del tumor sea eficaz, las células melanocíticas son capaces de interactuar con el microambiente que las rodea, en particular con la respuesta inmune del individuo a través de distintos mecanismos. ${ }^{10}$ La respuesta de las células $T$ ante células 
cancerígenas no difiere mucho a la respuesta normal a ciertos microorganismos. La activación de las células $\mathrm{T}$ es un proceso complejo, que inicia con la unión de receptores específicos en las células $\mathrm{T}$ (TCR) con el complejo péptido-MHC que se encuentra en la superficie de las células presentadoras de antígenos (APC). La activación absoluta de las células $T$ requiere de señales coestimuladoras en conjunto con señales coinhibidoras dadas por receptores de puntos de control inmunitario. La molécula CD28 es el mayor receptor coestimulador en las células $\mathrm{T}$, por medio de la interacción con los ligandos CD80 y CD86 en las APC, CD28 promueve la proliferación, producción de IL-2, y la supervivencia de las células T. Los puntos de control median la interacción de las células T "apagándolas" y previniendo la destrucción de células normales. Los receptores de puntos de control más estudiados son el antígeno 4 del linfocito $T$ citotóxico (CTLA-4) y receptor de muerte programada 1 (PD-1) presentes en la superficie de las células T y de las células T reguladoras. ${ }^{29}$ En la actualidad, estos receptores son parte de los objetivos en la inmunoterapia contra el melanoma avanzado (Figura 1).

El melanoma es una de las neoplasias capaces de regular la actividad de los puntos de control inmunitarios para así evitar su eliminación; a través de la expresión de PD-L1 y PD-L2 (ligandos de muerte celular programada 1 y 2 ) en las células tumorales, éstos actúan sobre el PD-1 de las células T, limitando su respuesta sobre el tejido tumoral. ${ }^{10}$

Por otro lado, la activación de CTLA-4 se logra a través de la unión con B7-1/B7-2 en la célula presentadora de antígeno; este mecanismo compite con la interacción de CD28:B7 inactivando a las células T. Los llamados inhibidores de puntos de control, en particular los bloqueadores de anticuerpos CTLA-4 y PD-1, han revolucionado el tratamiento con inmunoterapia en el melanoma, desafortunadamente no todos los casos con melanoma responden a estos tratamientos, además de sus múltiples efectos secundarios relacionados con el sistema inmune. ${ }^{29}$

Figura 1: Activación y control de respuestas de células T. Interacción del receptor de células T (TCR) y la molécula accesoria (CD4 o CD8) en la célula T con péptido-MHC en el APC, junto con la molécula coestimuladora CD28 en la célula T con CD80 o CD86 en el APC, que da como resultado la activación de la célula T. Los puntos de control inmunes CTLA-4 (naranja) y PD-1 (rojo) se expresan en las células T después de la activación y sirven para amortiguar las respuestas de las células T. Las células reguladoras también suprimen las funciones de las células T. CTLA-4 y PD-1 son objetivos de inmunoterapéuticos en melanoma. El bloqueo de estos puntos de control inmunes aumenta las respuestas antitumorales de las células $\mathrm{T}$. Modificado de: Ward WH et al. ${ }^{29}$

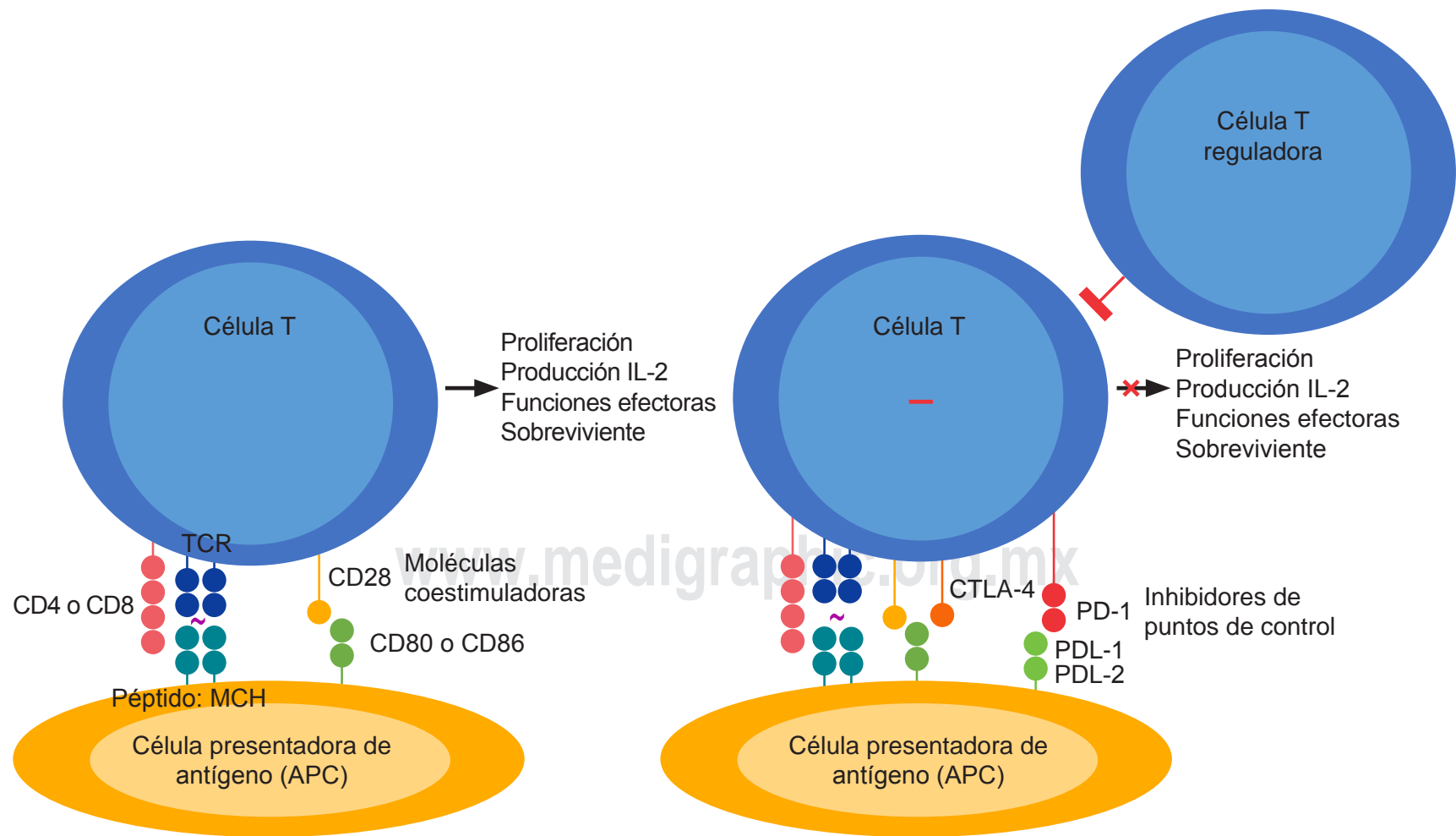




\section{INHIBIDOR CTLA-4}

El ipilimumab es un anticuerpo monoclonal humano del isotipo IgG1 que se une al punto de control CTLA-4, bloqueando la unión de sus ligandos, activando así a las células T y potenciando la respuesta inmunitaria. ${ }^{29}$ Dos ensayos controlados aleatorizadas en fase III con ipilimumab fueron realizados en personas con melanoma irresecable (estadio III-IV). En el primer ensayo se comparó el uso de ipilimumab con la vacuna péptido gp100, en donde se demostró una tasa de supervivencia de 10 meses con el uso de ipilimumab en comparación con seis meses con el uso de la vacuna péptido gp100. ${ }^{30}$ El segundo ensayo comparó el uso ipilimumab con dacarbazina y dacarbazina con placebo en personas con melanoma metastásico sin tratamiento previo, en el cual se observó una tasa de supervivencia de 12 y 9 meses respectivamente, con una mejoría en la supervivencia promedio (Tabla 1). ${ }^{31}$ Con base en estos resultados, en el 2011 la FDA aprobó el uso de ipilimumab como tratamiento para el melanoma irresecable (estadios III, IV); posteriormente se aprobó como terapia adyuvante en estadio III. ${ }^{29,32}$

\section{REACCIONES ADVERSAS DEL INHIBIDOR CTLA-4}

Dada la capacidad del ipilimumab para potenciar la respuesta de células $T$, su efecto es capaz de producir reacciones adversas relacionadas al sistema inmunológico. En los ensayos previos mencionados se evaluó la dosis de ipilimumab a 3 y $10 \mathrm{mg} / \mathrm{kg}$. En el primer ensayo los eventos adversos inmunológicos grado 3 (severo) y grado 4 (ame- naza la vida) se presentaron en $10-15 \%$ de los pacientes, ambos durante el periodo de inducción y reinducción; los sistemas más expuestos a dichos eventos fueron el gastrointestinal y piel, entre los efectos más frecuentes se observó diarrea en un 27 a 31\% de los pacientes tratados con ipilimumab. Los efectos residuales después de dos años de uso fueron colitis, prurito, erupción cutánea y vitíligo (1.5-2\%). Otros menos frecuentes incluyeron al sistema endocrino (hipotiroidismo, hipopituitarismo, hipofisitis e insuficiencia renal) y sistema hepático (hepatitis). Las muertes se asociaron a septicemia, perforación intestinal, falla orgánica múltiple, o síndrome de Guillain-Barre. Después de dicho ensayo, la FDA aprobó la dosis de ipilimumab para el melanoma a dosis de $3 \mathrm{mg} / \mathrm{kg}$ cada tres semanas con un total de cuatro dosis. ${ }^{30}$ En el segundo ensayo, en pacientes tratados con ipilimumab $(10 \mathrm{mg} / \mathrm{kg}$ ) y dacarbazina $(850 \mathrm{mg})$ los eventos adversos grado 3 y 4 se presentaron en el 56\%, en comparación con el $27 \%$ tratados con dacarbazina y placebo. ${ }^{31}$ La combinación de ipilimumab y dacarbazina no es ampliamente utilizada dado el porcentaje significativo de efectos adversos; sin embargo, la FDA aprobó su uso como tratamiento adyuvante en melanoma a dosis de 10 $\mathrm{mg}$ cada tres semanas cuatro dosis, seguido de $10 \mathrm{mg} / \mathrm{kg}$ cada 12 semanas por tres años o en el caso de enfermedad recurrente o toxicidad grave. ${ }^{31}$ Cabe mencionar que el uso de glucocorticoides sistémicos para el manejo de los efectos adversos no afecta la actividad antitumoral, esto sugiere que los mecanismos relacionados con el sistema inmune responsable de los efectos adversos inmunológicos son independientes a los de la respuesta antitumoral. ${ }^{33}$

Tabla 1: Resultados de ensayos clínicos en el tratamiento con ipilimumab en melanoma avanzado.

\begin{tabular}{|c|c|c|c|c|c|c|c|c|}
\hline Ref. & Agente & $n$ & $\begin{array}{c}\text { Tratamiento } \\
\text { previo }\end{array}$ & $\begin{array}{l}\text { Dosis de } \\
\text { ipilimumab }\end{array}$ & $\begin{array}{l}\text { Tasa de res- } \\
\text { puesta }(\%)\end{array}$ & $\begin{array}{l}\text { Promedio de } \\
\text { SLP (meses) }\end{array}$ & $\begin{array}{c}\text { Tasa de supervi- } \\
\text { vencia a } 1 \text { año (\%) }\end{array}$ & $\begin{array}{l}\text { EA grado } \\
3 \text { y } 4(\%)\end{array}$ \\
\hline \multirow[t]{2}{*}{30} & Ipilimumab & 131 & Sí & $\begin{array}{c}3 \mathrm{mg} / \mathrm{kg} \mathrm{c} / 3 \\
\text { sem } \times 4 \text { dosis* }\end{array}$ & 11 & $10.1^{* *}$ & 45.6 & $22.9^{* * *}$ \\
\hline & $\begin{array}{l}\text { Vacuna péptido } \\
\text { gp100 }\end{array}$ & 136 & & & 1.50 & $6.4^{* *}$ & 25.3 & $11.4^{\star \star \star}$ \\
\hline \multirow[t]{2}{*}{31} & $\begin{array}{l}\text { Ipilimumab + } \\
\text { dacarbazina }\end{array}$ & 250 & No & $\begin{array}{c}10 \mathrm{mg} / \mathrm{kg} \mathrm{c} / 3 \\
\text { sem } \times 4 \text { dosis* }\end{array}$ & 15.2 & $11.2^{* *}$ & $47.3^{\star \star}$ & 27 \\
\hline & $\begin{array}{l}\text { Placebo + } \\
\text { dacarbazina }\end{array}$ & 252 & & & 10.3 & 9.1 & 36.3 & 27.5 \\
\hline
\end{tabular}

EA = eventos adversos; Ref. = referencia; SLP = supervivencia libre de progresión.

* Dosis adicional para tasa de respuesta.

** Estadísticamente significativo.

${ }^{* * *}$ Adicionalmente hubo cuatro muertes en el grupo de ipilimumab, ocho muertes con ipilimumab + gp 100 , y dos muertes en el grupo solo con gp100.

Modificado de: Ward WH et al. ${ }^{29}$ 
Tabla 2: Resultados de ensayos clínicos en el tratamiento de melanoma avanzado.

\begin{tabular}{|c|c|c|c|c|c|c|c|}
\hline Ref. & Agente & $n$ & Dosis & $\begin{array}{c}\text { Tasa de } \\
\text { respuesta (\%) }\end{array}$ & $\begin{array}{l}\text { Promedio de } \\
\text { SLP (meses) }\end{array}$ & $\begin{array}{c}\text { Tasa de supervivencia } \\
\text { a } 1 \text { año }(\%)\end{array}$ & $\begin{array}{l}\text { EA grado } \\
3 \text { y } 4(\%)\end{array}$ \\
\hline \multirow[t]{2}{*}{35} & Nivolumab & 206 & $3 \mathrm{mg} / \mathrm{kg} \mathrm{c} / 2 \mathrm{sem}$ & 40 & $5.1^{*}$ & $72.9^{*}$ & $11.7^{*}$ \\
\hline & Dacarbazina & 205 & & 13.9 & 2.2 & $42.1^{*}$ & 17.6 \\
\hline \multirow[t]{3}{*}{37} & $\begin{array}{l}\text { Nivolumab + } \\
\text { Ipilimumab }\end{array}$ & 314 & $\begin{array}{c}1 \mathrm{mg} / \mathrm{kg} \mathrm{c} / 3 \mathrm{sem} \times 4 \\
\text { Después } 3 \mathrm{mg} / \mathrm{kg} \mathrm{c} / 2 \mathrm{sem} \\
3 \mathrm{mg} / \mathrm{kg} \mathrm{c} / 3 \mathrm{sem} \times 4\end{array}$ & $57.6^{*}$ & $11.5^{\star}$ & N/D & $55.0^{*}$ \\
\hline & Nivolumab & 316 & 3 mg/kg c/2 sem & $43.7^{\star}$ & 6.9 & & $16.3^{* *}$ \\
\hline & Ipilimumab & 315 & $3 \mathrm{mg} / \mathrm{kg} \mathrm{c} / 3 \mathrm{sem} \times 4$ & 19 & 2.9 & & $27.3^{\star \star}$ \\
\hline \multirow[t]{3}{*}{40} & Pembrolizumab & 278 & $10 \mathrm{mg} / \mathrm{kg} \mathrm{c} / 2 \mathrm{sem}$ & $33.7^{\star}$ & 5.5 & $74.1^{\star}$ & $13.3^{*}$ \\
\hline & Pembrolizumab & 277 & $10 \mathrm{mg} / \mathrm{kg} \mathrm{c} / 3 \mathrm{sem}$ & $32.9^{*}$ & 4.1 & $68.4^{*}$ & $10.1^{*}$ \\
\hline & Ipilimumab & 256 & $3 \mathrm{mg} / \mathrm{kg} \mathrm{c} / 3 \mathrm{sem} \times 4$ & $11.9^{*}$ & 2.8 & $58.2^{*}$ & $19.5^{*}$ \\
\hline
\end{tabular}

\section{INHIBIDOR PD-1}

Después de la introducción del ipilimumab, en el 2014 la FDA aprobó el uso de dos nuevos inhibidores de los puntos de control (pembrolizumab y nivolumab) como tratamiento de primera línea para el melanoma avanzado. Ambos fármacos bloquean la actividad de PD-1/PD-L1 restaurando así la actividad de las células $T$ y potenciando su respuesta. El nivolumab es un anticuerpo monoclonal humano del isotipo IgG4, el cual se une al receptor de muerte programada 1 (PD-1) y bloquea su interacción con sus ligandos (PD-L1 y PD-L2). ${ }^{29}$ En un ensayo controlado aleatorizado fase III en pacientes con melanoma en estadio IIIB-C y IV, en donde el ipilimumab e inhibidores de BRAF habían fallado, nivolumab demostró una respuesta de $31 \%$ en comparación con el uso de quimioterapia en donde la respuesta fue del $10 \% .{ }^{34} \mathrm{El}$ segundo ensayo en fase III se llevó a cabo en personas sin mutación BRAF en donde se comparó el uso de nivolumab vs dacarbazina, con una respuesta del $40 \%$ para nivolumab y $14 \%$ para dacarbazina, y una tasa de supervivencia al año de 73 y $43 \%$ respectivamente. ${ }^{35}$ Un tercer ensayo fase III se llevó a cabo en 905 pacientes con melanoma avanzado previamente resecado (estadio IIIB, C o IV), en donde se comparó la eficacia del nivolumab vs ipilimumab con un seguimiento mínimo por 18 meses, la tasa libre de recurrencia a un año fue de 70 y $60 \%$ respectivamente. ${ }^{36}$

Como se mencionó, los CTLA-4 y PD-1 suprimen la actividad de células T por vías inhibitorias distintas, así como en poblaciones distintas de células $\mathrm{T}$ y durante diferentes fases de la respuesta inmunológica, siendo esto objeto de interés para el uso combinado de ambos fármacos. ${ }^{29}$
En un estudio doble ciego con 142 pacientes con melanoma metastásico que no habían recibido tratamiento previo se evaluó la respuesta de ipilimumab $3 \mathrm{mg} / \mathrm{kg}$ combinado con nivolumab $1 \mathrm{mg} / \mathrm{kg}$ vs placebo una vez cada tres semanas, seguido de ipilimumab $3 \mathrm{mg} / \mathrm{kg}$, punto de corte para evaluar la respuesta entre pacientes con mutación BRAF V600. Se observó una respuesta en 61\% de los casos tratados con terapia combinada vs $11 \%$ en el grupo tratado con monoterapia. ${ }^{37}$ En un ensayo fase III en personas sin tratamiento previo, se comparó nivolumab e ipilimumab combinados, así como nivolumab e ipilimumab en monoterapia, donde se observó una tasa de respuesta de 57, 44 y 19\% respectivamente; una tasa de supervivencia de 11 meses para nivolumab e ipilimumab combinados vs siete meses para nivolumab y tres meses para ipilimumab en monoterapia. ${ }^{38}$ Después este mismo ensayo demostró una tasa de supervivencia a cinco años de $52 \%$ en el grupo combinado (60 meses), 44\% (36 meses) con nivolumab y $26 \%$ (20 meses) con ipilimumab. ${ }^{39} \mathrm{Ba}-$ sados en dicho estudios, la FDA aprobó el uso nivolumab como monoterapia en pacientes con melanoma avanzado, y en pacientes con mutación BRAF el uso combinado de nivolumab e ipilimumab. ${ }^{29}$

Un segundo inhibidor de PD-1 es el pembrolizumab. Al igual que el nivolumab, es un anticuerpo monoclonal del isotipo IgG4 que se une al PD-1 previniendo la interacción con su ligando. En un ensayo fase II en casos con melanoma avanzado, tratados previamente con ipilimumab e inhibidores BRAF o MEK, se comparó pembrolizumab a dosis de $2 \mathrm{mg} / \mathrm{kg}$ y $10 \mathrm{mg} / \mathrm{kg}$ cada tres semanas con el uso de quimioterapia, en donde la tasa libre de recurrencia 
fue $34 \%$ a dosis de $2 \mathrm{mg} / \mathrm{kg}, 38 \%$ a dosis de $10 \mathrm{mg} / \mathrm{kg}$ y $16 \%$ para el grupo con quimioterapia. ${ }^{38}$ En un ensayo aleatorizado fase III se comparó la dosis de pembrolizumab a dosis de $10 \mathrm{mg} / \mathrm{kg}$ cada dos y tres semanas con el uso de ipilimumab como tratamiento de primera línea, demostrando una tasa de supervivencia al año de 74 y $68 \%$ vs 58\% para ipilimumab; una tasa de respuesta del 33, 32 y $12 \%$ respectivamente. ${ }^{40}$ Después de los ensayos, la FDA aprobó el uso de pembrolizumab a dosis de $2 \mathrm{mg} / \mathrm{kg}$ cada tres semanas (Tabla 2). ${ }^{29}$ En el ensayo más reciente, con 514 pacientes con melanoma previamente resecado en estadio IIIA-C, se utilizó pembrolizumab a dosis de 200 mg cada tres semanas vs placebo en 505 personas, con una tasa libre de recurrencia al año de 75 y $61 \%$ respectivamente. ${ }^{41}$

\section{REACCIONES ADVERSAS DEL INHIBIDOR PDL-1}

En cuanto a los efectos adversos relacionados al sistema inmune, los inhibidores de PD-1 demuestran un patrón similar a los inhibidores de CTL-4. ${ }^{29}$ Los efectos adversos más frecuentes en casos tratados con inhibidores del punto de control PD-1 fueron fatiga, rash, diarrea, prurito y náuseas. ${ }^{34,35,37}$ La mayoría de los efectos adversos grados 3 y 4 se resolvieron con la suspensión del tratamiento y/o la administración de glucocorticoides sistémicos. ${ }^{34,35}$

Los efectos adversos relacionados al sistema inmune se presentaron en el $14 \%$ con el uso de nivolumab, en comparación con ipilimumab con 46\%; utilizados de manera combinada, los efectos adversos ocurrieron en el $59 \%$, con una media de resolución de los efectos menor a 12 semanas. ${ }^{35-37}$ Con respecto al uso de pembrolizumab en comparación con ipilimumab, los efectos adversos grados 3 y 5 con pembrolizumab se presentaron en el 10\% (cada dos semanas), 13\% (cada tres semanas) y $20 \%$ con ipilimumab..$^{40}$ En una revisión sistemática reciente se observó el riesgo de efectos adversos relacionados al sistema inmunológico, secundarios al uso de inhibidores de los puntos de control en el melanoma avanzado; los esquemas asociados con menores efectos fueron pembrolizumab 2 $\mathrm{mg} / \mathrm{kg}$ cada tres semanas; nivolumab $3 \mathrm{mg} / \mathrm{kg}$ cada dos semanas y pembrolizumab $10 \mathrm{mg} / \mathrm{kg}$ cada tres semanas; estos esquemas fueron los preferidos a comparación de otros utilizados. Por otro lado, hubo un incremento de los efectos adversos al utilizar nivolumab $1 \mathrm{mg} / \mathrm{kg}$ cada tres semanas en conjunto con ipilimumab $3 \mathrm{mg} / \mathrm{kg}$ cada tres semanas, por lo que este esquema debe ser utilizado con precaución. ${ }^{42}$

A pesar del éxito, sólo una porción de pacientes con melanoma avanzado responde a estos fármacos, con una taza de respuesta objetiva (OR) del $10-40 \%$ en monoterapia y hasta $58 \%$ con el uso combinado de nivolumab e ipilimumab. Debido a esto, la comunidad científica se ha centralizado en la identificación de ciertos marcadores biológicos que puedan ser identificados en personas con mayor probabilidad de éxito, así como en la identificación de caso con mayor probabilidad de fracaso, que puedan beneficiarse de tratamientos combinados más agresivos y así poder limitar los efectos adversos relacionados. ${ }^{29}$

\section{CONCLUSIÓN}

Este artículo responde a una revisión tradicional no sistematizada con el fin de contar con la información más actual hasta la fecha, tratándose de una patología de suma importancia y con un aumento considerable en nuestro país. Abordando desde los primeros indicios del conocimiento del melanoma, hasta abordar el objetivo principal de esta revisión: la inmunoterapia en estadios avanzados del melanoma. Debido a la continua evolución de los tratamientos específicos de control inmunitario y su inminente evolución con el paso de los años, el conocimiento de los últimos tratamientos para los pacientes con estados avanzados es prioritario, pues permite ampliar las opciones de tratamiento a pesar de contar con metástasis, sin olvidar que es necesario conocer los posibles efectos secundarios de la inmunoterapia. Como consecuencia de esto, se ha logrado prolongar la esperanza de vida, así como la calidad de vida en los últimos años de manera esperanzadora; el objetivo principal tiene y debe seguir siendo la prevención primaria, el aumento del conocimiento y educación por parte de la población mexicana sobre el cáncer de piel, debido a su inminente aumento durante los próximos años. Es de esperarse que aumenten las opciones terapéuticas, no sólo para el melanoma, sino en múltiples patologías, por lo que habrá un cambio constante en el paradigma, siendo de suma importancia el conocimiento de estas, motivo por el cual se fundamentó el actual trabajo.

\section{REFERENCIAS}

1. Rebecca VW, Sondak VK, Smalley KS. A brief history of melanoma: from mummies to mutations. Melanoma Res. 2012; 22 (2): 114-122.

2. Gershenwald JE, Guy GP Jr. Stemming the rising incidence of melanoma: calling prevention to action. J Natl Cancer Inst. 2015; 108 (1): djv381.

3. Parkin DM, Pisani P, Ferlay J. Estimates of the worldwide incidence of 25 major cancers in 1990. Int J Cancer. 1999; 80 (6): 827-841.

4. Ferlay J, Shin HR, Bray F, Forman D, Mathers C, Parkin DM. Estimates of worldwide burden of cancer in 2008: GLOBOCAN 2008. Int J Cancer. 2010; 127 (12): 2893-2917.

5. Melanoma Skin Cancer Statistics [Internet]. Cancer.org. 2020 [cited 17 April 2020]. Available from: https://www.cancer.org/cancer/ melanoma-skin-cancer/about/key-statistics.html

6. Global Cancer Observatory [Internet]. Gco.iarc.fr. 2018 [cited 17 April 2020]. Available from: http://gco.iarc.fr/

7. Martínez SH. El primer Consenso Nacional de Expertos en Melanoma. Gac Mex Oncol. 2005; 4 (2): 11-13.

8. Mort RL, Jackson IJ, Patton EE. The melanocyte lineage in development and disease. Development. 2015; 142 (4): 620-632. 
9. Bastian BC. The molecular pathology of melanoma: an integrated taxonomy of melanocytic neoplasia. Annu Rev Pathol. 2014; 9: 239-271.

10. Schadendorf D, Fisher DE, Garbe C, Gershenwald JE, Grob JJ, Halpern A et al. Melanoma. Nat Rev Dis Primers. 2015; 1: 15003.

11. Eggermont AM, Spatz A, Robert C. Cutaneous melanoma. Lancet. 2014; 383 (9919): 816-827.

12. Hawryluk EB, Tsao H. Melanoma: clinical features and genomic insights. Cold Spring Harb Perspect Med. 2014; 4 (9): a015388.

13. Ward KA, Lazovich D, Hordinsky MK. Germline melanoma susceptibility and prognostic genes: a review of the literature. J Am Acad Dermatol. 2012; 67 (5): 1055-1067.

14. Hodis E, Watson IR, Kryukov GV, Arold ST, Imielinski M, Theurillat JP et al. A landscape of driver mutations in melanoma. Cell. 2012; 150 (2): 251-263.

15. Huang FW, Hodis E, Xu MJ, Kryukov GV, Chin L, Garraway LA. Highly recurrent TERT promoter mutations in human melanoma. Science. 2013; 339 (6122): 957-959.

16. Horn S, Figl A, Rachakonda PS, Fischer C, Sucker A, Gast A et al. TERT promoter mutations in familial and sporadic melanoma. Science. 2013; 339 (6122): 959-961.

17. Krauthammer M, Kong Y, Ha BH, Evans P, Bacchiocchi A, McCusker JP et al. Exome sequencing identifies recurrent somatic RAC1 mutations in melanoma. Nat Genet. 2012; 44 (9): 1006-1014.

18. Shain AH, Bastian BC. From melanocytes to melanomas. Nat Rev Cancer. 2016; 16 (6): 345-358.

19. Carr S, Smith C, Wernberg J. Epidemiology and risk factors of melanoma. Surg Clin North Am. 2020; 100 (1): 1-12.

20. El Ghissassi F, Baan R, Straif K, Grosse Y, Secretan B, Bouvard V et al. A review of human carcinogens--part D: radiation. Lancet Oncol. 2009; 10 (8): 751-752.

21. IARC monographs on the evaluation of carcinogenic risks to humans. Solar and ultraviolet radiation. IARC Monogr Eval Carcinog Risks Hum. 1992; 55: 1-316.

22. Patel RV, Clark LN, Lebwohl M, Weinberg JM. Treatments for psoriasis and the risk of malignancy. J Am Acad Dermatol. 2009; 60 (6): 1001-1017.

23. Rastrelli M, Tropea S, Rossi CR, Alaibac M. Melanoma: epidemiology, risk factors, pathogenesis, diagnosis and classification. In Vivo. 2014; 28 (6): 1005-1011.

24. Markovic SN, Erickson LA, Rao RD, Weenig RH, Pockaj BA, Bardia A et al. Malignant melanoma in the 21st century, part 1: epidemiology, risk factors, screening, prevention, and diagnosis. Mayo Clin Proc. 2007; 82 (3): 364-380.

25. Gandini S, Sera F, Cattaruzza MS, Pasquini P, Abeni D, Boyle P et al. Meta-analysis of risk factors for cutaneous melanoma: I. Common and atypical naevi. Eur J Cancer. 2005; 41 (1): 28-44.

26. Goldman L, Schafer Al. Goldman-Cecil medicine. 26th ed. Philadelphia, PA: Elsevier/Saunders; 2019. 2 volumes (xl, 2722, I108 pages).

27. Cancer Statistics Review, 1975-2013-Previous Version-SEER Cancer Statistics Review [Internet]. SEER. 2020 [cited 17 April 2020]. Available from: https://seer.cancer.gov/archive/csr/1975_2013/.

28. Melanoma of the Skin-Cancer Stat Facts [Internet]. SEER. 2020 [cited 17 April 2020]. Available from: https://seer.cancer.gov/statfacts/html/ melan.html
29. Ward WH, Farma JM, editors. Cutaneous melanoma: etiology and therapy. Brisbane (AU): Codon Publications; 2017. doi: 10.15586/ codon.cutaneousmelanoma.2017

30. Hodi FS, O'Day SI, McDermott DF, Weber RW, Sosman JA, Haanen JB et al. Improved survival with ipilimumab in patients with metastatic melanoma. N Engl J Med. 2010; 363 (8): 711-723.

31. Robert C, Thomas L, Bondarenko I, O'Day S, Weber J, Garbe C et al. Ipilimumab plus dacarbazine for previously untreated metastatic melanoma. N Engl J Med. 2011; 364 (26): 2517-2526.

32. Eggermont AM, Chiarion-Sileni V, Grob JJ, Dummer R, Wolchok JD, Schmidt $\mathrm{H}$ et al. Prolonged survival in stage III melanoma with ipilimumab adjuvant therapy. N Engl J Med. 2016; 375 (19): 18451855.

33. Horvat TZ, Adel NG, Dang TO, Momtaz P, Postow MA, Callahan MK et al. Immune-related adverse events, need for systemic immunosuppression, and effects on survival and time to treatment failure in patients with melanoma treated with ipilimumab at memorial Sloan Kettering Cancer Center. J Clin Oncol. 2015; 33 (28): 3193-3198.

34. Weber JS, D'Angelo SP, Minor D, Hodi FS, Gutzmer R, Neyns B et al. Nivolumab versus chemotherapy in patients with advanced melanoma who progressed after anti-CTLA-4 treatment (CheckMate 037): a randomised, controlled, open-label, phase 3 trial. Lancet Oncol. 2015; 16 (4): 375-384.

35. Robert C, Long GV, Brady B, Dutriaux C, Maio M, Mortier L et al. Nivolumab in previously untreated melanoma without BRAF mutation. N Engl J Med. 2015; 372 (4): 320-330.

36. Weber J, Mandala M, Del Vecchio M, Gogas HJ, Arance AM, Cowey $\mathrm{CL}$ et al. Adjuvant nivolumab versus ipilimumab in resected stage III or IV melanoma. N Engl J Med. 2017; 377 (19): 1824-1835.

37. Larkin J, Chiarion-Sileni V, Gonzalez R, Grob JJ, Cowey CL, Lao CD et al. Combined nivolumab and ipilimumab or monotherapy in untreated melanoma. N Engl J Med. 2015; 373 (1): 23-34.

38. Ribas A, Puzanov I, Dummer R, Schadendorf D, Hamid O, Robert $C$ et al. Pembrolizumab versus investigator-choice chemotherapy for ipilimumab-refractory melanoma (KEYNOTE-002): a randomised, controlled, phase 2 trial. Lancet Oncol. 2015; 16 (8): 908-918.

39. Larkin J, Chiarion-Sileni V, Gonzalez R, Grob JJ, Rutkowski P, Lao $C D$ et al. Five-year survival with combined nivolumab and ipilimumab in advanced melanoma. N Engl J Med. 2019; 381 (16): 1535-1546.

40. Robert C, Schachter J, Long GV, Arance A, Grob JJ, Mortier L et al. Pembrolizumab versus ipilimumab in advanced melanoma. N Engl J Med. 2015; 372 (26): 2521-2532.

41. Eggermont AMM, Blank CU, Mandala M, Long GV, Atkinson V, Dalle $\mathrm{S}$ et al. Adjuvant pembrolizumab versus placebo in resected stage III melanoma. N Engl J Med. 2018; 378 (19): 1789-1801.

42. Chang CY, Park H, Malone DC, Wang CY, Wilson DL, Yeh YM et al. Immune checkpoint inhibitors and immune-related adverse events in patients with advanced melanoma: a systematic review and network meta-analysis. JAMA Netw Open. 2020; 3 (3): e201611. 